\title{
INFLUENCIA DEL LIDERAZGO DOCENTE EN LA EFECTIVIDAD DE LA ESCUELA DE POSGRADO DE LA UNE
}

\author{
INFLUENCE OF LEADERSHIP TEACHER IN THE EFFECTIVENESS OF THE GRADUATE \\ SCHOOL OF THE UNE
}

${ }^{1}$ Luis Eustaquio Huerta Camones

\begin{abstract}
Resumen
Esta investigación tuvo como propósito determinar la influencia del liderazgo que los docentes ejercen en las aulas sobre la efectividad de la escuela de posgrado de la UNE. Se basó en la teoría de liderazgo transformacional. Se usaron una muestra de 126 estudiantes, instrumentos adaptados y la regresión lineal múltiple. Los resultados más importantes encontrados fueron que el liderazgo docente influye positiva y significativamente en la efectividad de la escuela de posgrado de la UNE y que el efecto individual de las variables innovación docente, motivación docente y empatía docente sobre la efectividad de la escuela de posgrado de la UNE es positiva y significativa. Se concluye que el liderazgo docente en las aulas es un constructo importante por su influencia en los resultados institucionales y que para mejorar la efectividad de la escuela de posgrado de la UNE es necesario trabajar priorizando las variables empatía e innovación docente.
\end{abstract}

Palabras clave: liderazgo, transformacional, docente y efectividad.

\begin{abstract}
This research was to determine the influence of leadership that teacher perform in classroom on the effectiveness of graduate school UNE. This research was based on the theory of transformational leadership. A sample of 126 students, tools tailored and adapted, and multiple linear regression were used. The most important results were that teacher leadership positive and significantly influences the effectiveness of graduate school UNE, and the individual effect of the variables teacher innovation, teacher motivation and teacher empathy on the effectiveness of graduate school UNE is positive and significant. It is concluded that teacher leadership in the classroom is an important variable for their influence on organizational results, and to improve the effectiveness of graduate school UNE is necessary to work prioritizing the variables empathy and teaching innovation.
\end{abstract}

Keys words: leadership, transformational, teacher and effectiveness.

\section{Introducción}

La preocupación constante de que las universidades brinden un servicio educativo de mejor calidad, ante la exigencia social y los cambiantes nuevos tiempos, motiva a que se busque determinar las causas que explican los resultados institucionales. Es reconocido que siendo muchos los factores que explican dichos logros, el liderazgo docente es uno de ellos. Sin embargo, causa extrañeza que habiendo la teoría estudiado el liderazgo docente en el aula, sea aún un tópico poco conocido y difundido.

Según Pounder $(2006)^{2}$ el liderazgo docente es un tema de investigación reciente en la literatura sobre liderazgo

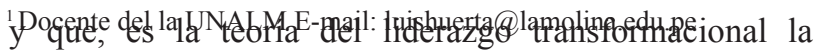

${ }^{2}$ Pounder, J. 2006. Transformational classroom leadership: The four wave of teacher leadership? Educational management administration leadership: 34; 533.
}

motivadora de dichas investigaciones.

Este trabajo, basándose en la teoría del liderazgo transformacional, busca determinar la influencia del liderazgo docente ejercida en las aulas sobre la efectividad institucional. También aspira ampliar la comprensión del liderazgo docente ejercida en las aula, para finalmente, proponer mejoras concretas al ejercicio del liderazgo de los docentes en las aulas de la escuela de posgrado de la UNE a fin de acrecentar los logros institucionales.

\section{Revisión de literatura}

El liderazgo y su efecto positivo en los logros organizacionales ha sido ampliamente aceptado tanto en el mundo práctico como el académico (Feng y Avery, $2008)^{3}$. Una expresión de esa aceptación es la existencia

${ }^{3}$ Feng, F. y Avery, G. 2008. Missing links in understanding the 
de una variedad importante de teorías que tratan sobre el liderazgo.

\section{Enfoques teóricos sobre el liderazgo}

Desde un punto de vista general y atendiendo a su impacto en la literatura, los enfoques teóricos sobre el liderazgo se pueden clasificar en positivista, conductual, contingencial y transformacional:

a. Desde el enfoque positivista, el liderazgo es definido a partir del conjunto de rasgos físicos, del carácter y la personalidad del líder. Estudios posteriores han demostrado que no existen pruebas científicas suficientes que respalden una teoría de liderazgo basada en rasgos.

b. Desde el enfoque conductual el liderazgo se define en función de la conducta. Las acciones individuales son realizadas por aquél que ostenta la autoridad y se centran en la toma de decisiones y el cumplimiento de las metas. Estudios experimentales, buscaron explorar los estilos autocrático, democrático y laissez faire. Se encontró que los líderes autocráticos son efectivos pero no tanto como los democráticos, aunque a estos les toma más tiempo alcanzar las metas debido a la participación colectiva. Los líderes laissez faire no lograban alcanzar las metas necesarias. Son también reconocidas las investigaciones realizadas en la Universidad del Estado de Ohio, que buscaron descubrir las dimensiones que caracterizan el comportamiento del líder. Los resultados permitieron identificar la consideración e iniciación de estructura como los factores que explican la mayor parte de la varianza. Del mismo modo, otros estudios interesantes en este enfoque son los realizados por la Universidad de Michigan, quienes se interesaron por establecer las diferencias de conductas que existen entre los líderes más y menos eficaces. En general, los resultados de los estudios de enfoque conductual han sido contradictorios e inconclusos, del mismo modo que tampoco se ha logrado un consenso sobre las categorías de conducta que son significativas.

c. El enfoque contingencial se fundamenta en la situación. Plantea que no hay un estilo mejor de liderazgo, que la eficacia de un estilo de liderazgo es contingente con algunos factores como las relaciones entre el líder y los miembros/seguidores, el clima o cultura organizacional, otros factores internos y ambientales o externos. Desde esta perspectiva, un solo estilo de liderazgo no puede ser aplicado en todas las situaciones cotidianas de una organización ya que varía de acuerdo a las situaciones particulares. Algunos reconocidos modelos y teorías situaciones son: la Teoría de Tannenbaum y Schmidt: El continúo de la conducta del líder, el Modelo Contingencial de Fiedler, la Teoría de Path - Goal de House y Mitchell, el Modelo de Stinson y Jhonson, el Modelo Contingencial de

relationship between leadership and organizational performance. International Business \& Economics Research Journal, Vol. 7, Num. 5.
Vroom e Yetton, la Teoría Tridimensional de Hersey y Blanchard, el Modelo Integrativo de la Conducta Eficaz de Dirección, entre otras. De modo general, la mayor contribución de las teorías contingenciales es la inclusión de variables situacionales moderadoras, las cuales son variadas en unas y otras. En algunas teorías, la gran cantidad de variables hace que el modelo no sea parsimonioso lo que contribuye a que sea mucho más difícil probar. Asimismo, muchas de ellas incluyen variables intervinientes para explicar cómo los líderes influyen en los subordinados.

d. La primera teoría del enfoque transformacional comienza con Kouzes y Poster (Mendoza, 2005), quienes al observar con detenimiento el proceso dinámico del liderazgo mediante el análisis de casos y encuestas, reconocieron cinco prácticas fundamentales a través de las cuales los líderes logran realizar cosas extraordinarias. El modelo de liderazgo cobró vida, carácter y color cuando empezaron a escucharse historias de personas corrientes que obtenían logros fuera de lo común. Encontraron que los líderes estudiados era capaces de realizar las cinco prácticas siguientes: desafiar los procesos, inspirar una visión compartida, habilitar a los demás para que actúen, modelar el camino y dar aliento al corazón.

\section{Teo ría del liderazgo transformacional y transaccional}

Actualmente Bass y Avolio son reconocidos como los precursores de un modelo de liderazgo que responde a las exigencias del siglo XXI. Sus estudios se han convertido en una teoría sólida con evidencia empírica en diversos países y culturas. EL modelo de liderazgo transformacional y transaccional de dichos autores responde de manera directa a los nuevos enfoques administrativos que contemplan temas tales como: la calidad, la innovación organizacional, la planeación estratégica creativa, la orientación hacia los procesos organizacionales entre otros. Es un enfoque que contempla aspectos relacionados con la transformación de los individuos y las organizaciones.

Mendoza (2006) 5 menciona que, Bass y Avolio proponen el modelo de liderazgo de rango completo (Full Range Leadership FRL), el cual incluye los componentes del liderazgo transformacional, transaccional y no liderazgo.

\section{Liderazgo transformacional:}

Según Bass y Avolio (2002), "el conocimiento de los factores contextuales que típicamente afectan a los equipos, puede ser útil a los líderes para ayudarles a

\footnotetext{
4 Mendoza, Ignacio. 2005. Estudio diagnóstico del perfil de liderazgo transformacional y transaccional de gerentes de ventas de una empresa farmacéutica a nivel nacional. Tesis para optar el grado de Doctor en Ciencias Administrativas de la Universidad Autónoma de Tlaxcalá. México.

5 Mendoza, Martha. 2006. Liderazgo transformacional, dimensiones e impacto en la cultura organizacional y eficacia de las empresas. Revista de la Facultad de Ciencias Económicas de la Universidad Autónoma del Estado de México.
} 
entender y vencer los obstáculos organizacionales que impiden el desarrollo eficaz del grupo". Entre los aspectos del contexto que afectan el funcionamiento de los equipos, los autores señalan a la cultura como el fundamental. Por tanto, el liderazgo debe orientar sus acciones a su construcción y el estilo de liderazgo transformacional contribuye a ello mediante comportamientos orientados a fortalecer la cultura organizacional y comportamientos orientados a la forma de la cultura organizacional.

Para el establecimiento de esta nueva cultura, se requiere que el líder estimule intelectualmente a los colaboradores a fin de que puedan redefinir sus valores y clarificar la misión. Para lo cual también se requiere del carácter inspiracional del líder transformacional mediante la visión y articulación de un estado futuro deseado o mediante el desarrollo de una visión de futuro con su equipo.

Otro de los aspectos que enfatiza el liderazgo transformacional es la consideración individual. En una organización los niveles de interés, las formas de participar, las capacidades, las formas de percibir difieren de un miembro a otro. Las habilidades cognitivas, es decir, las características particulares de los individuos que incluyen el conocimiento, estrategias y habilidades que adquiere mediante la educación o la experiencia, son tenidas en cuenta por el líder a la hora de asignar las tareas. De modo que los miembros más capaces apoyen a los menos capaces y de esta manera alcanzar un alto desempeño. De igual modo el líder considera los rasgos no cognitivos como la personalidad, los intereses, actitudes y valores toda vez que estos rasgos afectan la motivación, el desempeño, el compromiso y la lealtad. El rol más importante del líder transformacional es el de inspirar al equipo y a los individuos mediante la realización de tareas desafiantemente significativas y de suma importancia para la organización.

No obstante, esa forma individual que tienen cada uno de percibir los problemas y de contribuir a la solución de los mismos puede ser modificada por el líder mediante la estimulación intelectual (enfrentar los problemas de manera creativa) y la motivación inspiracional. Por lo tanto, el líder debe tener una idea de las fortalezas y debilidades de los miembros del equipo para comprender cómo dichas características pueden afectar al funcionamiento del equipo y reconocer que cada contribución individual puede estar influenciada por las características del líder, el estilo de liderazgo, así como las características de la tarea. El líder transformacional busca, asimismo, "crear en los miembros una conciencia de equipo que permite valorar los resultados y trascender las necesidades individuales"

En suma, este estilo de liderazgo resulta ser un proceso recíproco en el que los miembros de la una comunidad construyen significados que guían propósitos comunes. Esto entraña el establecimiento de acuerdos consensuados, estímulos, orientación abierta y permanente, como también, la participación activa y democrática en que esté implicado un compromiso de todos para ejecutar responsablemente las acciones orientadas a la transformación de la organización.

Las dimensiones del liderazgo transformacional son: carisma, estimulación intelectual, consideración individualizada y motivación inspiracional.

\section{Liderazgo Transaccional:}

El otro liderazgo identificado por Bass es el transaccional. En este tipo el líder actúa de dos maneras: promete y da recompensas (reconocimiento pecuniario y refuerzos) en función del esfuerzo realizado y del nivel de rendimiento conseguido por los subalternos (recompensas contingenciales), e interviene corrigiendo ante la omisión de un deber o cuando no se han alcanzado los niveles deseados (dirección por excepción). El líder transaccional se rige por valores como honestidad, responsabilidad, imparcialidad y la rectitud en los compromisos.

A diferencia del liderazgo transformacional que se centra en el autoconcepto y la autoestima, el líder transaccional, al orientarse a las necesidades materiales de los colaboradores, obtiene niveles mucho más bajos de resultados (Bass, 1999).

En suma, los líderes transaccionales suelen ser buenos negociadores, autoritarios y, en ocasiones hasta, agresivos. Necesitan ser competentes en aquellos aspectos que se refieren a la organización de las personas y los recursos para conseguir los objetivos de una manera eficiente. Este líder desarrolla competencias como la gestión de los recursos, dirección de personas, gestión del tiempo y toma de decisiones.

\section{No liderazgo - dejar hacer - laissez faire:}

Otro de los estilos de liderazgo propuesto por Bass es el No Liderazgo (laissez faire). Este estilo hace referencia al líder que evita tomar decisiones, no se implica, no se define, toma distancia de las situaciones que requieren necesariamente de su presencia y que son cruciales para la institución. Los resultados del estudio, realizado por Pascual y colaboradores, indican que la ausencia de liderazgo, sobre todo en los momentos difíciles, lleva al profesorado a percibir un mayor abandono, y por consiguiente, una mayor ineficiencia del director. "La conducta directiva de "Dejar hacer" provoca altos niveles de insatisfacción en los profesores, lo que se traduce en un desaliento laboral" (Pascual y otros, 1993).

\section{Liderazgo transformacional del docente}

Pounder (2006) señala que la noción del docente líder en las investigaciones comenzó a cobrar fuerza desde los 80 s. Este concepto ha pasado por diferentes estadios o concepciones hasta llegar finalmente a considerar a las actividades cotidianas que realiza el docente en el aula y

\footnotetext{
Pacual, R.; Villa, A.; Auzmendi, E. 1993. El liderazgo transformacional en los centros docentes. Un estudio en las comunidades autónomas del País Vasco y Castilla-León. Director de la colección: Aurelio Villa. Bilbao: ICE Universidad de Deusto. Ediciones Mensajero.
} 
frente a sus estudiantes como las acciones propias de un líder. La primera concepción acerca del liderazgo de los docentes está asociada con la clásica figura jerárquica del docente director o jefe de los otros docentes. Se destaca del papel formal del docente jefe de otros docentes.

La segunda concepción está más asociada al liderazgo instruccional del docente. Es decir, líderes formales que participan, por ejemplo, como jefes de equipo para mejorar el currículo y supervisores de los otros docentes. Estos papeles son producto del sistema escolar jerárquico por lo que su concepción es administrativa y formal. Normalmente, estos docentes se dedican en forma parcial a la administración sin dejar su labor docente en las aulas.

La tercera concepción enfatiza el liderazgo del docente más allá de una posición jerárquica o formal. Implica un cambio importante en la concepción pues introduce la idea de que el liderazgo docente es un proceso más que una posición. Resalta que el docente al realizar sus labores cotidianas está ejerciendo el liderazgo. Más precisamente, se puede llamar líder docente al que, fuera de los marcos formales, coordina actividades entre los docentes para mejorar aspectos pedagógicos, ayudan a rediseñar la institución, ayudan a resolver problemas institucionales, etc. Se involucra en forma personal y voluntaria. En este particular caso, a estos docentes se les puede llamar líderes transformacionales. Del mismo modo, un líder es reconocido porque es también excelente en su labor de docente en el aula y es un modelo debido precisamente a su efectiva enseñanza. La razón de ello estaría en que el docente expresa sus características de líder transformacional en el aula logrando acrecentar los resultados educativos. Entonces, los buenos docentes los serían gracias a su habilidad o cualidades de líderes transformacionales. Inversamente, los buenos líderes transformacionales gracias a sus propias características serían excelentes docentes. Existen evidencias de que las características de líder transformacional podrían ayudar a ser pedagógicamente excelentes (Crowter, 1997)7. Entonces podemos decir, que un gran líder es un gran docente.

La cuarta concepción reconocería el papel de líder docente liderando a sus estudiantes en el aula. Esta función adicional del docente es importante porque permitiría mejorar la formación del estudiante. Esa formación exige no sólo una labor pedagógica sino de liderazgo, como una característica altamente valorada, a fin de buscar que los estudiantes, por ejemplo, desarrollen las habilidades de liderazgo para poder ejercerlas en su vida estudiantil y sobre todo profesional. Las universidades, con mayor razón en el posgrado, han de formar a profesionales que se conviertan en líderes sociales y de opinión, consejeros, asesores o emprendedores (Atencio y Arrieta, 2005) ${ }^{8}$.

\footnotetext{
Crowther, F. 1997. Teachers as leaders. An exploratory framework, International journal of educational management 11(1): 6-13.

8 Atencio, L. y Arrieta, B. 2005. El liderazgo y la toma de decisiones en las organizaciones de educación superior. Omnia, Vol. 11, n ${ }^{\circ}$ 1, pp. $1-24$
}

Dimensiones del liderazgo transformacional:

Las dimensiones del liderazgo transformacional docente, no serían las propuestas por el enfoque transformacional debido a su generalidad. En ese sentido no abundan las escalas o instrumentos específicos que puedan ser de utilidad para los docentes. Diversos autores sugieren que es necesario contar con un mayor conocimiento específico del liderazgo del docente en las aulas y se recomienda para ello, perfeccionar las escalas de liderazgo actual, desarrollando procedimientos más sencillos para facilitar la toma de decisiones en el aula (Moss y Ritossa, 2007).

Cerda y Ramírez (2010) ${ }^{9}$, luego de hacer una revisión exhaustiva y fundamentados en la teoría del liderazgo transformacional, proponen que el constructo liderazgo docente en el aula, sea configurado en tres grandes áreas: creatividad/experimentación (desarrollo de nuevos enfoques para viejos problemas), motivación docente (capacidad de entusiasmar, transmitir confianza y respeto) y empatía docente (tratar individualmente al estudiante). Esta propuesta coincide ampliamente con las nuevas corrientes más estrictamente pedagógica que sostienen y defienden tres ámbitos de actuación del profesor efectivo: disciplinar (el profesor como innovador y cuestionador del conocimiento), pedagógico (capacidad del docente de transmitir motivación y entusiasmo en su actividad) y personal (como tutor de la adquisición autónoma del conocimiento por los estudiantes).

\section{Efectividad}

Según Cervera $(2010)^{10}$, la idea fundamental del estudio de la efectividad organizacional es que se trata de una forma de evaluar a las organizaciones, pero con la particularidad de que es una evaluación social, o sea, que la hacen aquellos que tienen algún tipo de relación con ella, que bien pueden ser investigadores interesados en el tema, el público en general o simples observadores.

Bajo esta perspectiva se puede decir que efectividad es un constructo construido por los diferentes participantes organizacionales que demuestran interés en la organización, y quienes la evalúan haciendo juicios subjetivos.

Es importante resaltar que el concepto de efectividad es diferente a los conceptos de eficacia y eficiencia organizacional, y que algunas se confunden y su aplicación no es la correcta. La eficacia se refiere a la capacidad de la organización de lograr sus metas, y la eficiencia es la relación entre los productos obtenidos y los recursos empleados. La efectividad en cambio, puede incluir a ambos conceptos, y se puede dar el caso de que una organización sea eficaz y eficiente, pero no efectiva desde el punto de vista de sus participantes.

\footnotetext{
Cerdá, M. y Ramírez, M. 2010. Evaluación de los estilos de liderazgo en la docencia: una aplicación en la enseñanza universitaria de postgrado de la Universidad Autónoma de Baja California, México.

${ }^{10}$ Cervera, Fernando. 2010. La percepción de la efectividad organizacional. Recuperado el 20/03/2012 de http://octi.guanajuato.gob.mx/sinnco/ formulario/MT/MT2010/MT12/SESION3/MT123

FCERVERAS_203.pdf
} 


\section{Relación entre el liderazgo transformacional y la efectividad organizacional}

Feng y Avery (2008), hacen una exhaustiva revisión de publicaciones científicas analizando la relación entre el liderazgo y los resultados organizacionales. En forma resumida, señalan que si bien el tema ha sido ampliamente tratado en estos 25 años revisados, no existen evidencias concluyentes sobre el impacto del liderazgo en la eficacia organizacional y una razón de ello sería que hace falta mejorar en la parte conceptual y metodológica de las investigaciones, habida cuenta que ni siquiera un concepto de liderazgo ha logrado un amplio acuerdo hasta nuestros días. Sin embargo, también reconoce que a nivel general es ampliamente aceptado que el liderazgo ejerce un efecto en los logros organizacionales y que existe consenso sobre la contribución importante que ha hecho la teoría del liderazgo transformacional, destacándose sobre las más antiguas y sobre otras más actuales teorías.

\section{Método}

Sobre la metodología se puede decir que, aún cuando la mayoría de estudios revisados sólo emplea la correlación para estudiar la relación entre el liderazgo y la eficacia organizacional, también hay estudios donde se utiliza la regresión lineal. Por ejemplo, Ruiz $(2011)^{11}$, en un estudio realizado en instituciones educativas secundarias, encuentra que el $\mathrm{R}^{2}$ del efecto del liderazgo transformacional en la eficacia institucional es de $57 \%$ y que la influencia es positiva y significativa. También Pedraza (2004) $)^{12}$, en un estudio realizado en organizaciones que pertenecen a la administración pública, encuentra que su modelo de los estilos de liderazgo explica el 51\% de las variaciones en la eficacia de dichas organizaciones. En ambos casos se utilizaron mediciones subjetivas o escala de opiniones.

\section{Objetivos:}

El objetivo general es determinar la influencia del liderazgo docente en la efectividad de la escuela de posgrado de la UNE. Los objetivos específicos son determinar la influencia de la innovación docente en la efectividad de la escuela de posgrado de la UNE, determinar la influencia de la motivación docente en la efectividad de la escuela de posgrado de la UNE y determinar la influencia de la empatía docente en la efectividad de la escuela de posgrado de la UNE.

\section{Hipótesis:}

La hipótesis general es que el liderazgo docente influye significativamente en la efectividad de la escuela de la UNE. Las hipótesis específicas son que la innovación docente influye significativamente en la efectividad de

\footnotetext{
${ }^{11}$ Ruiz, G. 2011. Influencia del estilo de liderazgo del director en la eficacia de las instituciones educativas del consorcio "Santo Domingo de Guzmán" de Lima Norte. Tesis de maestría, Universidad Nacional Mayor de San Marcos, Lima, Perú.

${ }^{12}$ Pedraza, L. 2004. Efectos del estilo de liderazgo sobre la eficacia de las prganizaciones públicas. Revista facultad de ingeniería, Vol. $12 \mathrm{~N}^{\circ} 2$.
}

la escuela de posgrado de la UNE, que la motivación docente influye significativamente en la efectividad de la escuela de posgrado de la UNE y que la empatía docente influye significativamente en la efectividad de la escuela de posgrado de la UNE.

\section{Variables:}

La variable independiente, liderazgo docente, está conformado por las dimensiones innovación docente, motivación docente y empatía docente. Ella mide la percepción de los estudiantes sobre las acciones que realizan sus docentes en las actividades cotidianas de sus clases. La variable dependiente, efectividad de la escuela de posgrado de la UNE, se mide por el puntaje obtenido en un cuestionario. Este capta la percepción de los estudiantes sobre la efectividad general de la escuela de posgrado de la UNE.

Diseño:

Se adaptaron cuestionarios y se aplicaron a los estudiantes en los que se recogió sus percepciones, tanto sobre la conducta que sus respectivos docentes muestran en sus aulas como del desempeño general de la escuela de posgrado de la UNE. Los cuestionarios se construyeron con la escala de likert de 5 opciones. Las pruebas de hipótesis se realizaron mediante la regresión lineal múltiple. Previamente, se hicieron las verificaciones de cumplimiento de los supuestos para trabajar con la estadística paramétrica y se evalúa la presencia de multicolinealidad. Se utilizó una muestra, hallada mediante la fórmula de muestras finitas, conformada por 126 estudiantes de la escuela de posgrado de la UNE en el año 2013 y se procesaron mediante el SPSS 20.

Los criterios para clasificar las percepciones de los estudiantes a cada una de las variables son:

Cuadro 1. Criterios De Valoración.

\begin{tabular}{cc}
\hline Rango de valores & Clasificación \\
\hline $1.00-2.30$ & Bajo \\
$2.40-3.60$ & Regular \\
$3.70-5.00$ & Alto \\
\hline
\end{tabular}

\section{Resultados y discusión}

Resultados descriptivos

Cuadro 2. Resultados descriptivos.

\begin{tabular}{cccc}
\hline & $\mathrm{N}$ & Media & Desv. típ. \\
\hline Innovación & 126 & 3,159 &, 83448 \\
Motivación & 126 & 3,559 &, 81480 \\
Empatía & 126 & 3,254 &, 78929 \\
Efectividad & 126 & 3,506 &, 77467 \\
N válido & 126 & & \\
\hline
\end{tabular}


El promedio de todas las variables está ligeramente por encima de la media tres. En general, los estudiantes valoran como regular a todas las variables de estudio.

En forma más detallada se puede observar que la motivación docente es la que tiene una mayor valoración y la innovación docente tiene la menor valoración. Que la motivación sea la más alta estaría reflejando el hecho de que los estudiantes perciben que los docentes los motivan, les hablan con entusiasmo y les proporcionan elogios apropiados. En otras palabras, el vínculo emocional que establecen los docentes con sus estudiantes es elevado.

El siguiente en valoración es la efectividad. Es decir, los estudiantes perciben que la escuela está alcanzando las metas esperadas. Esto en general refleja la percepción de que la escuela de posgrado de la UNE aún mantiene su prestigio en la calidad de sus egresados. Un poco menos valora está la empatía de los docentes hacia sus alumnos. De acuerdo a las preguntas del cuestionario estaría reflejando, comparado con las dos anteriores variables, una menor atención individualizada y un reducido vínculo personal con el estudiante. Por último, la menor valoración es para las prácticas docentes innovadoras en el aula. Es decir, los docentes realizan escasas innovaciones en sus métodos y materiales didácticos así como escasamente fomentan la emergencia de nuevas ideas o nuevas soluciones a los problemas.

\section{Resultados inferenciales}

Confiabilidad de los instrumentos: las variables innovación docente, motivación docente y empatía docente presentan una fuerte confiabilidad con alfas de Cronbach mayores a 0,787 . Mientras que la variable efectividad de la escuela muestra una alta confiabilidad con un alfa de Cronbach mayor de 0,92. En suma se puede afirmar que el requisito de la confiabilidad está satisfecho.

Normalidad de los datos: dado que la significación de todas las variables es mayor a 0,05 se puede inferir que no existen razones suficientes para rechazar la hipótesis nula de normalidad. Es decir, la distribución de los datos es normal en cada uno de los casos analizados.

Prueba de multicolinealidad: considerando que se usa la regresión lineal múltiple para hacer las pruebas de hipótesis, es necesario probar que las variables independientes no presenten problemas de multicolinealidad. Una primera forma de probarlo es a través de la correlación entre las variables:
Cuadro 3. Correlaciones entre variables

\begin{tabular}{|c|c|c|c|c|}
\hline & & Innovación & Motivación & Empatía \\
\hline \multirow{3}{*}{ Innovación } & $\begin{array}{l}\text { Correlación } \\
\text { de Pearson }\end{array}$ & 1 &, 644 & ,643 \\
\hline & $\begin{array}{c}\text { Sig. } \\
\text { (bilateral) }\end{array}$ & &, 000 &, 000 \\
\hline & $\mathrm{N}$ & 126 & 126 & 126 \\
\hline \multirow{3}{*}{ Motivación } & $\begin{array}{l}\text { Correlación } \\
\text { de Pearson }\end{array}$ & ,644 & 1 &, 598 \\
\hline & $\begin{array}{c}\text { Sig. } \\
\text { (bilateral) }\end{array}$ &, 000 & &, 000 \\
\hline & $\mathrm{N}$ & 126 & 126 & 126 \\
\hline \multirow{3}{*}{ Empatía } & $\begin{array}{l}\text { Correlación } \\
\text { de Pearson }\end{array}$ & ,643 &, 598 & 1 \\
\hline & $\begin{array}{c}\text { Sig. } \\
\text { (bilateral) }\end{array}$ & ,000 &, 000 & \\
\hline & $\mathrm{N}$ & 126 & 126 & 126 \\
\hline
\end{tabular}

La regla práctica dice que si ninguna de las correlaciones es mayor que 0,70 se puede descartar la posibilidad de multicolinealidad entre las variables. En el caso de este estudio, la más alta correlación es de 0,64. Lo que indicaría ausencia de multicolinealidad.

Otra manera de probar la existencia de multicolinealidad es a través de la prueba FIV (factor de inflación de la varianza):

Cuadro 4. Estadísticos de colinealidad.

\begin{tabular}{ccc}
\hline Modelo & Tolerancia & FIV \\
\hline (Constante) & & \\
Innovación &, 481 & 2,078 \\
Motivación &, 528 & 1,895 \\
Empatía &, 528 & 1,895 \\
\hline
\end{tabular}

La regla, citada por Kleinbraum, Kupper y Muller (1988), indica que existen problemas de multicolinealidad si algún FIV es superior a 10, que corresponde a un $\mathrm{R}^{2}=$ 0,90 y a una Tolerancia $<0,1$. En nuestro caso, el FIV más alto es de 2,07 y la menor tolerancia es 0,481. Esta prueba también permite descartar problemas de multicolinealidad.

\section{Estimación del modelo de regresión}

Efectividad $=\alpha+\beta_{1}$ Innovación $+\beta_{2}$ Motivación $+\beta_{3}$ Empatía $+\epsilon$

Cuadro 5. Resumen del modelo.

\begin{tabular}{ccccc}
\hline Modelo & $\mathrm{R}$ & $\mathrm{R}$ cuadrado & $\begin{array}{c}\text { R cuadrado } \\
\text { corregida }\end{array}$ & $\begin{array}{c}\text { Error } \\
\text { típ. de la } \\
\text { estimación }\end{array}$ \\
\hline 1 &, $737^{\mathrm{a}}$ &, 543 &, 532 & 2,650 \\
\hline a. Variables predictoras: (Constante), Empatía, Motivación, Innovación
\end{tabular}


El resumen del modelo indica que las variables innovación, motivación y empatía docente explican un $54 \%$ de la variabilidad de la efectividad de la escuela. Como es conocido, un valor mayor al $50 \%$ en ciencias sociales indica una buena asociación lineal entre las variables y que el modelo es suficientemente apropiado para realizar explicaciones y predicciones.

Cuadro 6. ANOVA $^{\mathrm{a}}$

\begin{tabular}{ccccccc}
\hline Modelo & $\begin{array}{c}\text { Suma de } \\
\text { cuadrados }\end{array}$ & Gl & $\begin{array}{c}\text { Media } \\
\text { cuadrática }\end{array}$ & F & Sig. \\
\hline & Regresión & $1,018,383$ & 3 & 339,461 & 48,325 &, $000^{\mathrm{b}}$ \\
1 & Residual & 856,990 & 122 & 7,025 & & \\
& Total & $1,875,373$ & 125 & & & \\
\hline
\end{tabular}

a. Variable dependiente: Efectividad

b. Variables predictoras: (Constante), Empatía, Motivación, Innovación

El análisis de la varianza muestra una $\mathrm{F}$ con sig. 0,000 . Esto nos permite rechazar la hipótesis nula de que la variabilidad observada en la variable respuesta (efectividad de la escuela) sea explicada por el azar y podemos admitir que hay asociación entre la variable dependiente y las independientes. En otras palabras, una F con sig. 0.000 implica que el modelo de regresión sirve para explicar la variable dependiente en función de las independientes.

\section{Cuadro 7. Coeficientes ${ }^{\mathrm{a}}$}

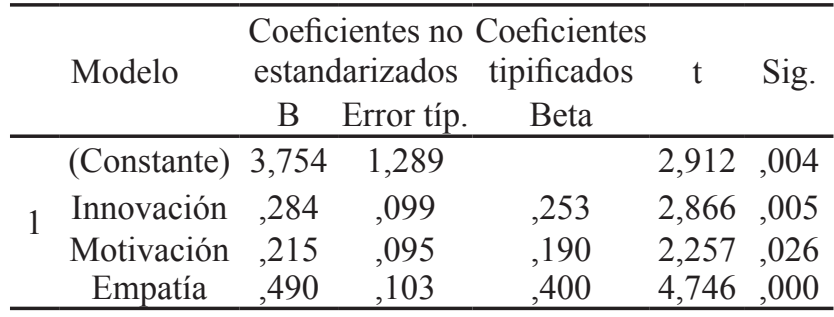

a. Variable dependiente Efectividad.

Los resultados de cada uno de los coeficientes $\beta$ s indican el cambio promedio en la variable dependiente (efectividad de la escuela) cuando una de las variables predictoras o independientes (innovación, motivación o empatía) cambia en una unidad adicional asumiendo que las otras permanecen constantes. Podemos notar que todos los coeficientes de las variables independientes son positivos y con un sig. menor a 0,05 . Asimismo, la variable que tiene un mayor impacto en la efectividad de la escuela es la empatía docente, luego viene la innovación docente y por último la motivación docente.

\section{PRUEBA DE HIPÓTESIS}

\section{Hipótesis Específicas}

Hipótesis Específicas 1: La innovación docente influye significativamente en la efectividad de la escuela de posgrado de la UNE.

$$
\begin{aligned}
& \mathrm{H}_{0}: \beta_{1}=0 \\
& \mathrm{H}_{\mathrm{a}}: \beta_{\mathrm{l}} \neq 0
\end{aligned}
$$

En el cuadro 7 , podemos notar que que $\beta_{1}$ toma el valor de 0,284 y que la prueba t tiene asociado un sig. 0,005. Eso implica que existen razones suficientes para rechazar la hipótesis nula y aceptar que la innovación docente influye positiva y significativamente en la efectividad de la escuela de posgrado de la UNE.

Hipótesis Específicas 2: La motivación docente influye significativamente en la efectividad de la escuela de posgrado de la UNE.

$$
\begin{aligned}
& \mathrm{H}_{0}: \beta_{2}=0 \\
& \mathrm{H}_{\mathrm{a}}: \beta_{2} \neq 0
\end{aligned}
$$

En el cuadro 7, podemos notar que $\beta_{2}$ toma el valor de 0,215 y que la prueba $t$ tiene asociado un sig. 0,026 . Ello implica que existen razones suficientes para rechazar la hipótesis nula y aceptar que la motivación docente influye positiva y significativamente en la efectividad de la escuela de posgrado de la UNE.

Hipótesis Específicas 3: La empatía docente influye significativamente en la efectividad de la escuela de posgrado de la UNE.

$$
\begin{aligned}
& \mathrm{H}_{0} \beta_{3}=0 \\
& \mathrm{H}_{\mathrm{a}}: \beta_{3} \neq 0
\end{aligned}
$$

En el cuadro 7, podemos notar que $\beta_{3}$ toma el valor de 0,490 y que la prueba t tiene asociado un sig. 0,000. Ello implica que existen razones suficientes para rechazar la hipótesis nula y aceptar que la empatía docente influye positiva y significativamente en la efectividad de la escuela de posgrado de la UNE.

\section{Hipótesis general:}

El liderazgo docente (innovación, motivación y empatía en forma conjunta) influye significativamente en la efectividad de la escuela de la UNE.

$\mathrm{H}_{0}: \beta_{1}=\beta_{2}=\beta_{3}=0$

$\mathrm{H}_{\mathrm{a}}$ : Al menos uno de los coeficientes es diferente de cero.

En el cuadro de la ANOVA, podemos observar que la prueba $\mathrm{F}$ tiene asociado un sig. 0,000. Ello implica que existen razones suficientes para rechazar la hipótesis nula y que por lo tanto, el liderazgo docente (en al menos una de las variables innovación, motivación o empatía) influye significativamente en la efectividad de la escuela de posgrado de la UNE. Además si observamos en el cuadro de los coeficientes, todos los $\beta$ s tienen signo positivo y tienen asociado un sig. menor a 0,05. Es decir, se observa que todos los coeficientes son positivos 
y significativamente diferentes de cero. Con lo que queda suficientemente rechazada la hipótesis nula y se puede afirmar que el liderazgo docente, conformado por la innovación, motivación y empatía, influye significativamente en la efectividad de la escuela de posgrado de la UNE.

\section{El modelo identificado es:}

$\begin{array}{ll}\text { Efectividad de } & 3,754+0,284 \text { Innovación docente }+ \\ \text { la escuela }= & 0,215 \text { Motivación docente }+0,490 \\ & \text { Empatía docente }\end{array}$

\section{Conclusiones}

Se encuentra que el modelo propuesto tiene un nivel apropiado de explicación al presentar una $\mathrm{R}^{2}$ de 0,543. Destacándose adicionalmente que, ese resultado es concordante con los que hallan Ruiz (2011) y su $\mathrm{R}^{2}$ de 0.57 y también con el de Pedraza (2004) y su $\mathrm{R}^{2}$ de 0.51 . Asimismo, también se halla que el liderazgo docente (medido por las variables innovación, motivación y empatía) influye positiva y significativamente en la eficacia de la escuela de posgrado de la UNE. Al mismo tiempo, se demuestra que el efecto individual de las variables innovación, motivación y empatía sobre la efectividad de la escuela es positiva y significativa. Resaltándose que de todas ellas, las variables más importantes son la empatía y la innovación docente. Es decir, para mejorar la efectividad de la escuela de posgrado de la UNE, se tendría que poner énfasis en trabajar las dos preferentemente porque sus impactos son mayores que el de la motivación. En otras palabras, se puede afirmar que se ha explicitado un reto importante para la escuela de posgrado de la UNE, porque en los resultados descriptivos la variable más valorada (tiene una mayor presencia) es la motivación (el vínculo emocional de los docentes con los alumnos) mientras que el modelo causal propone trabajar preferentemente con la empatía e innovación. Es decir, los alumnos están manifestando que hay suficiente vínculo emocional entre docente y alumnos pero se carece de un vínculo empático y de innovación didáctica y de nuevas maneras de resolver los problemas. Finalmente, para un mejoramiento sostenido y a largo plazo, se recomienda capacitar a los docentes en la mejora de sus habilidades de construir relaciones empáticas y de poder realizar permanentes innovaciones pedagógicas y, sería destacable, realizar seguimientos permanentes o estudios periódicos para poder evaluar los cambios en la percepción de los alumnos sobre sus docentes y su escuela.

\section{Literatura citada}

Bass, B. 1999. Two decades of research and development in transformational leadership. European journal of work and organizational psychology, 8 (1), 9-32.

Bass, B. and Avolio, B. 2002. Improving organizational effectiveness through transformational leadership. Edit.

\section{Sage Press: Estados Unidos.}

Moss, S. and Ritossa, D. 2007. The Impact of Goal Orientation on the Association between Leadership Style and Follower Performance, Creativity and Work Attitudes. Leadership, Vol. 3, no 4, pp. 433-456. 